\title{
Efficient Quantum Tensor Product Expanders and $k$-designs
}

\author{
Aram W. Harrow \\ Department of Mathematics, University of Bristol, Bristol, U.K. \\ Richard A. Low* \\ Department of Computer Science, University of Bristol, Bristol, U.K.
}

February 24, 2019

\begin{abstract}
We give an efficient construction of constant-degree, constant-gap quantum $k$-tensor product expanders. The key ingredients are an efficient classical tensor product expander and the quantum Fourier transform. Our construction works whenever $k=O(n / \log n)$, where $n$ is the number of qubits. An immediate corollary of this result is an efficient construction of approximate unitary $k$-designs on $n$ qubits for any $k=O(n / \log n)$.
\end{abstract}

\section{Introduction}

Random unitaries are a key resource in quantum computation and information. They find algorithmic uses (e.g. [12]) and cryptographic applications (e.g. 3, 7]). However, a simple parameter counting argument shows that a Haar-random unitary cannot be implemented efficiently. Instead, we will seek to construct efficient pseudo-random ensembles of unitaries which resemble the Haar measure for certain applications. For example, a $k$-design (often referred to as a $t$ design, or a $(k, k)$-design) is a distribution on unitaries which matches the first $k$ moments of the Haar distribution. Below, we will give an efficient construction of a $k$-design for any $k$. We will do this by first finding an efficient construction of a quantum ' $k$-copy tensor product expander' (defined later), which can then be iterated to produce a $k$-design. We will therefore need to understand some of the theory of expanders before presenting our construction.

Classical expander graphs have the property that a marker executing a random walk on the graph will be close to uniformly distributed after a small number of steps. We consider a generalisation of this, due to [6], to graphs that randomise $k$ different markers carrying out correlated random walks on the same graph. This is a stronger requirement than for a normal $(k=1)$ expander because the correlations between walkers (unless they start at the same position) must be broken. We then generalise quantum expanders in the same way, so that the unitaries act on $k$ copies of the system. We give an efficient construction of a quantum ' $k$-copy tensor product expander' (TPE or $k$-TPE) which uses an efficient classical $k$-TPE as its main ingredient. We then give as a key application the first efficient construction of a unitary $k$-design for any $k$.

While randomised constructions yield $k$-designs (by a modification of Theorem 5 of [1]) and $k$-TPEs (when the dimension is polynomially larger than $k$ [6]) with near-optimal parameters, these approaches are not efficient. State $k$-designs, meaning ensembles of quantum states

\footnotetext{
*low@cs.bris.ac.uk
} 
matching the first $k$ moments of the uniform distribution on pure states, have been efficiently constructed in [2, but their approach does not appear to generalise to (unitary) $k$-designs. Previous efficient constructions of $k$-designs were known only for $k=1,2$, and no efficient constant-degree, constant-gap quantum $k$-TPEs were previously known, except for the $k=1$ case corresponding to quantum expanders.

In Sec. 1.1, we will define quantum expanders and other key terms. Then in Sec. 1.2 we will describe our main result which will be proved in Sec. 2.

\subsection{Quantum Expanders}

If $\mathcal{S}_{N}$ denotes the symmetric group on $N$ objects and $\pi \in \mathcal{S}_{N}$, then define

$$
B(\pi):=\sum_{i=1}^{N}|\pi(i)\rangle\langle i|
$$

to be the matrix that permutes the basis states $|1\rangle, \ldots,|N\rangle$ according to $\pi$.

We will only consider $D$-regular expander graphs here. We can think of a random walk on such a graph as selecting one of $D$ permutations of the vertices randomly at each step. We construct the permutations as follows. Label the vertices from 1 to $N$. Then label each edge from 1 to $D$ so that each edge label appears exactly once on the incoming and outgoing edges of each vertex. This gives a set of $D$ permutations. We can take a random walk on the graph by applying a uniformly chosen one of those $D$ permutations, although below we will consider more general probability distributions.

We now define a classical $k$-TPE:

Definition 1.1 ([6]). Let $\nu$ be a probability distribution on $\mathcal{S}_{N}$ with support on $\leq D$ permutations. Then $\nu$ is an $(N, D, \lambda, k)$ classical $k$-copy tensor product expander if

$$
\left\|\mathbb{E}_{\pi \sim \nu}\left[B(\pi)^{\otimes k}\right]-\mathbb{E}_{\pi \sim \mathcal{S}_{N}}\left[B(\pi)^{\otimes k}\right]\right\|_{\infty}=\left\|\sum_{\pi \in \mathcal{S}_{N}}\left(\nu(\pi)-\frac{1}{N !}\right) B(\pi)^{\otimes k}\right\|_{\infty} \leq \lambda .
$$

with $\lambda<1$. Here $\mathbb{E}_{\pi \sim \nu}$ means the expectation over $\pi$ drawn according to $\nu$ and $\mathbb{E}_{\pi \sim \mathcal{S}_{N}}$ means the expectation over $\pi$ drawn uniformly from $\mathcal{S}_{N}$.

Setting $k=1$ recovers the usual spectral definition of an expander.

The degree of the map is $D=|\operatorname{supp} \nu|$ and the gap is $1-\lambda$. Ideally, the degree should be small and gap large. To be useful, these should normally be independent of $N$ and possibly $k$. We say that a TPE construction is efficient if it can be implemented in poly $\log N$ steps. There are known constructions of efficient classical TPEs. The construction of Hoory and Brodsky [8] provides an expander with $D=$ poly $\log N$ and $\lambda=1-1 / \operatorname{poly}(k, \log N)$ with efficient running time. An efficient constant-degree construction is also known, due to Kassabov [9, which has constant degree and gap (independent of $N$ and $k$ ).

Similarly, we define a quantum $k$-TPE. First we introduce the notation

$$
U^{\otimes k, k}=U^{\otimes k} \otimes\left(U^{*}\right)^{\otimes k} .
$$

Definition $1.2([6])$. Let $\nu$ be a distribution on $\mathcal{U}(N)$, the group of $N \times N$ unitary matrices, with $D=|\operatorname{supp} \nu|$. Then $\nu$ is an $(N, D, \lambda, k)$ quantum $k$-copy tensor product expander if

$$
\left\|\mathbb{E}_{U \sim \nu}\left[U^{\otimes k, k}\right]-\mathbb{E}_{U \sim \mathcal{U}(N)}\left[U^{\otimes k, k}\right]\right\|_{\infty} \leq \lambda
$$


with $\lambda<1$. Here $\mathbb{E}_{U \sim \mathcal{U}(N)}$ means the expectation over $U$ drawn from the Haar measure.

Again, normally we want $D$ and $\lambda$ to be constants and setting $k=1$ recovers the usual definition of a quantum expander. Note that an equivalent statement of the above definition is that, for all $\rho$,

$$
\left\|\mathbb{E}_{U \sim \nu}\left[U^{\otimes k} \rho\left(U^{\dagger}\right)^{\otimes k}\right]-\mathbb{E}_{U \sim \mathcal{U}(N)}\left[U^{\otimes k} \rho\left(U^{\dagger}\right)^{\otimes k}\right]\right\|_{2} \leq \lambda\|\rho\|_{2}
$$

A natural application of this is to make an efficient unitary $k$-design. A unitary $k$-design is the same as a quantum $k$-TPE except is close in the 1 -norm rather than the $\infty$-norm:

Definition 1.3. Let $\nu$ be a distribution on $\mathcal{U}(N)$ with $D=|\operatorname{supp} \nu|$. Say that $\nu$ is an $\epsilon$ approximate unitary $k$-design if

$$
\left\|\mathbb{E}_{U \sim \nu}\left[U^{\otimes k, k}\right]-\mathbb{E}_{U \sim U(N)}\left[U^{\otimes k, k}\right]\right\|_{1} \leq \epsilon .
$$

As for TPEs, we say that a unitary design is efficient if a poly $\log (N)$-time algorithm exists to sample $U$ from $\nu$ and to implement $U$.

Other definitions of approximate designs are possible; for example we can use the diamond norm [10] between the superoperators $\hat{\mathcal{E}}_{\mathcal{U}(N)}^{k}$ and $\hat{\mathcal{E}}_{\nu}^{k}$ where

$$
\hat{\mathcal{E}}_{\mathcal{U}(N)}^{k}(\rho)=\mathbb{E}_{U \sim \mathcal{U}(N)}\left[U^{\otimes k} \rho\left(U^{\dagger}\right)^{\otimes k}\right]
$$

and

$$
\hat{\mathcal{E}}_{\nu}^{k}(\rho)=\mathbb{E}_{U \sim \nu}\left[U^{\otimes k} \rho\left(U^{\dagger}\right)^{\otimes k}\right]
$$

We can then, following [5], define an $\epsilon$-approximate $k$-design as a set of unitaries $\mathcal{U}$ with

$$
\left\|\hat{\mathcal{E}}_{\mathcal{U}(N)}^{k}-\hat{\mathcal{E}}_{\nu}^{k}\right\|_{\diamond} \leq \epsilon .
$$

While these norms are in general incomparable, our results work efficiently for both definitions and indeed for any norms that are related by a factor that is polynomial in dimension.

We can make an $\epsilon$-approximate unitary $k$-design from a quantum $k$-TPE with $O(k \log N)$ overhead:

Theorem 1.4. If $\mathcal{U}$ is an $(N, D, \lambda, k)$ quantum $k$-TPE then iterating the map $m=\frac{1}{\log 1 / \lambda} \log \frac{N^{2 k}}{\epsilon}$ times gives an $\epsilon$-approximate unitary $k$-design with $D^{m}$ unitaries.

Proof. Iterating the TPE $m$ times gives

$$
\left\|\mathbb{E}_{U \sim \nu}\left[U^{\otimes k, k}\right]-\mathbb{E}_{U \sim \mathcal{U}(N)}\left[U^{\otimes k, k}\right]\right\|_{\infty} \leq \lambda^{m}
$$

This implies that

$$
\left\|\mathbb{E}_{U \sim \nu}\left[U^{\otimes k, k}\right]-\mathbb{E}_{U \sim \mathcal{U}(N)}\left[U^{\otimes k, k}\right]\right\|_{1} \leq N^{2 k} \lambda^{m}
$$

We take $m$ such that $N^{2 k} \lambda^{m}=\epsilon$ to give the result.

Corollary 1.5. A construction of an efficient quantum $(N, D, \lambda, k)$-TPE yields an efficient approximate unitary $k$-design, provided $\lambda=1-1 /$ poly $\log N$. Further, if $D$ and $\lambda$ are constants, the number of unitaries in the design is $N^{(O(k))}$. 
Our approach to construct an efficient quantum $k$-TPE will be to take an efficient classical $2 k$-TPE and mix it with a quantum Fourier transform. The degree is thus only larger than the degree of the classical expander by one. Since the quantum Fourier transform on $\mathbb{C}^{N}$ requires poly $\log (N)$ time, it follows that if the classical expander is efficient then the quantum expander is as well. The main technical difficulty is to show for suitable values of $k$ that the gap of the quantum TPE is not too much worse than the gap of the classical TPE.

A similar approach to ours was first used in [6] to construct a quantum expander by mixing a classical 2-TPE with a phase. However, regardless of the set of phases chosen, this approach will not yield quantum $k$-TPEs from classical $2 k$-TPEs for any $k \geq 2$.

\subsection{Main Result}

Let $\omega=e^{2 \pi i / N}$ and define the $N$-dimensional Fourier transform to be $\mathcal{F}=\frac{1}{\sqrt{N}} \sum_{m=1}^{N} \sum_{n=1}^{N} \omega^{m n}|m\rangle\langle n|$. Define $\delta_{\mathcal{F}}$ to be the distribution on $\mathcal{U}(N)$ consisting of a point mass on $\mathcal{F}$. Our main result is that mixing $\delta_{\mathcal{F}}$ with a classical $2 k$-TPE yields a quantum $k$-TPE for appropriately chosen $k$ and $N$.

Theorem 1.6. Let $\nu_{C}$ be a classical $\left(N, D, 1-\epsilon_{C}, 2 k\right)-T P E$, and for $0<p<1$, define $\nu_{Q}=$ $p \nu_{C}+(1-p) \delta_{\mathcal{F}}$. Suppose that

$$
\epsilon_{A}:=1-2(2 k)^{4 k} / \sqrt{N}>0 .
$$

Then $\nu_{Q}$ is a quantum $\left(N, D+1,1-\epsilon_{Q}, k\right)$-TPE where

$$
\epsilon_{Q} \geq \frac{\epsilon_{A}}{12} \min \left(p \epsilon_{C}, 1-p\right)>0
$$

The bound Eqn.1.10 is optimised when $p=1 /\left(1+\epsilon_{C}\right)$, in which case we have

$$
\epsilon_{Q} \geq \frac{\epsilon_{A} \epsilon_{C}}{24}
$$

This means that any constant-degree, constant-gap classical expander gives a quantum expander with constant degree and gap. If the the classical expander is efficient then the quantum expander is as well. Unfortunately the construction does not work for all dimensions, only dimensions with $N=\Omega\left((2 k)^{8 k}\right)$, so that $\epsilon_{A}$ is lower-bounded by a positive constant. However, in applications normally $k$ is fixed. An interesting open problem is to find a construction that works for all dimensions, in particular a $k=\infty$ expander. We believe our construction may work for $k$ as large as $c N$ for a small constant $c$. On the other hand, if $2 k>N$ then the gap in our construction drops to zero.

\section{Proof of Theorem 1.6}

\subsection{Proof overview}

First, we introduce some notation. Define $\mathcal{E}_{\mathcal{S}_{N}}^{2 k}=\mathbb{E}_{\pi \sim \mathcal{S}_{N}}\left[B(\pi)^{\otimes 2 k}\right]$ and $\mathcal{E}_{\mathcal{U}(N)}^{k}=\mathbb{E}_{U \sim \mathcal{U}(N)}\left[U^{\otimes k, k}\right]$. These are both projectors onto spaces which we label $V_{\mathcal{S}_{N}}$ and $V_{\mathcal{U}(N)}$ respectively. Since $V_{\mathcal{U}(N)} \subset$ $V_{\mathcal{S}_{N}}$, it follows that $\mathcal{E}_{\mathcal{S}_{N}}^{2 k}-\mathcal{E}_{\mathcal{U}(N)}^{k}$ is a projector onto the space $V_{0}:=V_{\mathcal{S}_{N}} \cap V_{\mathcal{U}(N)}^{\perp}$. We also define $\mathcal{E}_{\nu_{C}}^{2 k}=\mathbb{E}_{\pi \sim \nu_{C}}\left[B(\pi)^{\otimes 2 k}\right]$ and $\mathcal{E}_{\nu_{Q}}^{k}=\mathbb{E}_{U \sim \nu_{Q}}\left[U^{\otimes k, k}\right]$. 
The idea of our proof is to consider $\mathcal{E}_{\nu_{C}}^{2 k}$ a proxy for $\mathcal{E}_{\mathcal{S}_{N}}^{2 k}$; if $\lambda_{C}$ is small enough then this is a reasonable approximation. Then we can restrict our attention to vectors in $V_{0}$, which we would like to show all shrink substantially under the action of our expander. This in turn can be reduced to showing that $\mathcal{F}^{\otimes k, k}$ maps any vector in $V_{0}$ to a vector that has $\Omega(1)$ amplitude in $V_{S_{N}}^{\perp}$. This last step is the most technically involved step of the paper, and involves careful examination of the different vectors making up $V_{\mathcal{S}_{N}}$.

Thus, our proof reduces to two key Lemmas. The first allows us to substitute $\mathcal{E}_{\nu_{C}}^{2 k}$ for $\mathcal{E}_{\mathcal{S}_{N}}^{2 k}$ while keeping the gap constant.

Lemma 2.1 ([6] Lemma 1). Let $\Pi$ be a projector and let $X$ and $Y$ be operators such that $\|X\|_{\infty} \leq 1,\|Y\|_{\infty} \leq 1, \Pi X=X \Pi=\Pi,\|(I-\Pi) X(I-\Pi)\|_{\infty} \leq 1-\epsilon_{C}$ and $\|\Pi Y \Pi\|_{\infty} \leq 1-\epsilon_{A}$. Assume $0<\epsilon_{C}, \epsilon_{A}<1$. Then for any $0<p<1,\|p X+(1-p) Y\|_{\infty}<1$. Specifically,

$$
\|p X+(1-p) Y\|_{\infty} \leq 1-\frac{\epsilon_{A}}{12} \min \left(p \epsilon_{C}, 1-p\right) .
$$

We will restrict to $V_{\mathcal{U}(N)}^{\perp}$, or equivalently, subtract the projector $\mathcal{E}_{\mathcal{U}(N)}^{k}$ from each operator. Thus we have $X=\mathcal{E}_{\nu_{C}}^{2 k}-\mathcal{E}_{\mathcal{U}(N)}^{k}, \Pi=\mathcal{E}_{\mathcal{S}_{N}}^{2 k}-\mathcal{E}_{\mathcal{U}(N)}^{k}$ and $Y=\mathcal{F}^{\otimes k, k}-\mathcal{E}_{\mathcal{U}(N)}^{k}$. According to Definition 1.1, we have the bound

$$
\|(I-\Pi) X(I-\Pi)\|_{\infty}=\left\|\mathcal{E}_{\nu_{C}}^{2 k}-\mathcal{E}_{\mathcal{S}_{N}}^{2 k}\right\|_{\infty} \leq 1-\epsilon_{C} .
$$

It will remain only to bound $\lambda_{A}:=1-\epsilon_{A}=\left\|\left(\mathcal{E}_{\mathcal{S}_{N}}^{2 k}-\mathcal{E}_{\mathcal{U}(N)}^{k}\right) \mathcal{F}^{\otimes k, k}\left(\mathcal{E}_{\mathcal{S}_{N}}^{2 k}-\mathcal{E}_{\mathcal{U}(N)}^{k}\right)\right\|_{\infty}$.

Lemma 2.2. For $N \geq(2 k)^{2}$,

$$
\lambda_{A}=\left\|\left(\mathcal{E}_{\mathcal{S}_{N}}^{2 k}-\mathcal{E}_{\mathcal{U}(N)}^{k}\right) \mathcal{F}^{\otimes k, k}\left(\mathcal{E}_{\mathcal{S}_{N}}^{2 k}-\mathcal{E}_{\mathcal{U}(N)}^{k}\right)\right\|_{\infty} \leq 2(2 k)^{4 k} / \sqrt{N} .
$$

Combining Eqn. 2.2, Lemma 2.2 and Lemma 2.1 now completes the proof of Theorem 1.6.

\subsection{Action of a Classical $2 k$-TPE}

We start by analysing the action of a classical $2 k$-TPE. (We consider $2 k$-TPEs rather than general $k$-TPEs since our quantum expander construction only uses these.) The fixed points are states which are unchanged when acted on by $2 k$ copies of any permutation matrix. Since the same permutation is applied to all copies, any equal indices will remain equal and any unequal indices will remain unequal. This allows us to identify the fixed points of the classical expander: they are the sums over all states with the same equality and difference constraints. For example, for $k=1$ (corresponding to a 2-TPE), the fixed points are $\sum_{n_{1}}\left|n_{1}, n_{1}\right\rangle$ and $\sum_{n_{1} \neq n_{2}}\left|n_{1}, n_{2}\right\rangle$ (all off-diagonal entries equal to 1 ). In general, there is a fixed point for each partition of the set $\{1,2, \ldots, 2 k\}$ into at most $N$ non-empty parts. If $N \geq 2 k$, which is the only case we consider, the $2 k^{\text {th }}$ Bell number $\beta_{2 k}$ gives the number of such partitions (see e.g.[13]).

We now write down some more notation to further analyse this. If $\Pi$ is a partition of $\{1, \ldots, 2 k\}$, then we write $\Pi \vdash 2 k$. We will see that $\mathcal{E}_{\mathcal{S}_{N}}^{2 k}$ projects onto a space spanned by vectors labeled by partitions. For a partition $\Pi$, say that $(i, j) \in \Pi$ if and only if elements $i$ and $j$ are in the same block. Now we can write down the fixed points of the classical expander. Let

$$
I_{\Pi}=\left\{\left(n_{1}, \ldots, n_{2 k}\right): n_{i}=n_{j} \text { iff }(i, j) \in \Pi\right\} .
$$

This is a set of tuples where indices in the same block of $\Pi$ are equal and indices in different blocks are not equal. The corresponding state is

$$
\left|I_{\Pi}\right\rangle=\frac{1}{\sqrt{\left|I_{\Pi}\right|}} \sum_{\mathbf{n} \in I_{\Pi}}|\mathbf{n}\rangle
$$


where $\mathbf{n}=\left(n_{1}, \ldots, n_{2 k}\right)$ and $|\Pi|$ is the number of blocks in $\Pi$. Note that the $\left\{I_{\Pi}\right\}_{\Pi \vdash 2 k}$ form a partition $\{1, \ldots, N\}^{2 k}$ and thus the $\left\{\left|I_{\Pi}\right\rangle\right\}_{\Pi \vdash 2 k}$ form an orthonormal basis for $V_{\mathcal{S}_{N}}$. This is because, when applying the same permutation to all indices, indices that are the same remain the same and indices that differ remain different. This implies that

$$
\mathcal{E}_{\mathcal{S}_{N}}^{2 k}=\sum_{\Pi \vdash 2 k}\left|I_{\Pi}\right\rangle\left\langle I_{\Pi}\right|
$$

To evaluate the normalisation, use $\left|I_{\Pi}\right|=(N)_{|\Pi|}$ where $(N)_{n}$ is the falling factorial $N(N-$ $1) \ldots(N-n+1)$. We will later find it useful to bound $(N)_{n}$ with

$$
\left(1-\frac{n^{2}}{2 N}\right) N^{n} \leq(N)_{n} \leq N^{n}
$$

We will also make use of the refinement partial order:

Definition 2.3. The refinement partial order $\leq$ on partitions $\Pi, \Pi^{\prime} \in \operatorname{Par}(2 k, N)$ is given by

$$
\Pi \leq \Pi^{\prime} \text { iff }(i, j) \in \Pi \Rightarrow(i, j) \in \Pi^{\prime} .
$$

For example, $\{\{1,2\},\{3\},\{4\}\} \leq\{\{1,2,4\},\{3\}\}$. Note that $\Pi \leq \Pi^{\prime}$ implies that $|\Pi| \geq\left|\Pi^{\prime}\right|$.

\subsubsection{Turning Inequality Constraints into Equality Constraints}

In the analysis, it will be easier to consider just equality constraints rather than both inequality and equality constraints as in $I_{\Pi}$. Therefore we make analogous definitions:

$$
E_{\Pi}=\left\{\left(n_{1}, \ldots, n_{2 k}\right): n_{i}=n_{j} \forall(i, j) \in \Pi\right\}
$$

and

$$
\left|E_{\Pi}\right\rangle=\frac{1}{\sqrt{\left|E_{\Pi}\right|}} \sum_{\mathbf{n} \in E_{\Pi}}|\mathbf{n}\rangle .
$$

Then $\left|E_{\Pi}\right|=N^{|\Pi|}$. For $E_{\Pi}$, indices in the same block are equal, as with $I_{\Pi}$, but indices in different blocks need not be different.

We will need relationships between $I_{\Pi}$ and $E_{\Pi}$. First, observe that $E_{\Pi}$ can be written as the union of some $I_{\Pi}$ sets:

$$
E_{\Pi}=\bigcup_{\Pi^{\prime} \geq \Pi} I_{\Pi^{\prime}}
$$

To see this, note that for $\mathbf{n} \in E_{\Pi}$, we have $n_{i}=n_{j} \forall(i, j) \in \Pi$, but we may also have an arbitrary number of additional equalities between $n_{i}$ 's in different blocks. The (unique) partition $\Pi^{\prime}$ corresponding to these equalities has the property that $\Pi$ is a refinement of $\Pi^{\prime}$; that is, $\Pi^{\prime} \geq \Pi$. Thus for any $\mathbf{n} \in E_{\Pi}$ there exists a unique $\Pi^{\prime} \geq \Pi$ such that $\mathbf{n} \in I_{\Pi^{\prime}}$. Conversely, whenever $\Pi^{\prime} \geq \Pi$, we also have $I_{\Pi^{\prime}} \subseteq E_{\Pi^{\prime}} \subseteq E_{\Pi}$ because each inclusion is achieved only be relaxing constraints.

Using Eqn. 2.11, we can obtain a useful identity involving sums over partitions:

$$
N^{|\Pi|}=\left|E_{\Pi}\right|=\sum_{\Pi^{\prime} \geq \Pi}\left|I_{\Pi^{\prime}}\right|=\sum_{\Pi^{\prime} \geq \Pi} N_{\left(\left|\Pi^{\prime}\right|\right)} .
$$

Additionally, since both sides in Eqn. 2.12 are degree $|\Pi|$ polynomials and are equal on $\geq|\Pi|+1$ points (we can choose any $N$ in Eqn. 2.12 with $N \geq 2 k$ ), it implies that $x^{|\Pi|}=\sum_{\Pi^{\prime} \geq \Pi} x_{\left(\Pi^{\prime}\right)}$ as an identity on formal polynomials in $x$. 
The analogue of Eqn. 2.11 for the states $\left|E_{\Pi}\right\rangle$ and $\left|I_{\Pi}\right\rangle$ is similar but has to account for normalisation factors. Thus we have

$$
\sqrt{\left|E_{\Pi}\right|}\left|E_{\Pi}\right\rangle=\sum_{\Pi^{\prime} \geq \Pi} \sqrt{\left|I_{\Pi^{\prime}}\right|}\left|I_{\Pi^{\prime}}\right\rangle
$$

We would also like to invert this relation, and write $\left|I_{\Pi}\right\rangle$ as a sum over various $\left|E_{\Pi^{\prime}}\right\rangle$. Doing so will require introducing some more notation. Define $\zeta\left(\Pi, \Pi^{\prime}\right)$ to be 1 if $\Pi \leq \Pi^{\prime}$ and 0 if $\Pi \leq \Pi^{\prime}$. This can be thought of as a matrix that, with respect to the refinement ordering, has ones on the diagonal and is upper-triangular. Thus it is also invertible. Define $\mu\left(\Pi, \Pi^{\prime}\right)$ to be the matrix inverse of $\zeta$, meaning that for all $\Pi_{1}, \Pi_{2}$, we have

$$
\sum_{\Pi^{\prime} \vdash 2 k} \zeta\left(\Pi_{1}, \Pi^{\prime}\right) \mu\left(\Pi^{\prime}, \Pi_{2}\right)=\sum_{\Pi^{\prime} \vdash 2 k} \mu\left(\Pi_{1}, \Pi^{\prime}\right) \zeta\left(\Pi^{\prime}, \Pi_{2}\right)=\delta_{\Pi_{1}, \Pi_{2}},
$$

where $\delta_{\Pi_{1}, \Pi_{2}}=1$ if $\Pi_{1}=\Pi_{2}$ and $=0$ otherwise. Thus, if we rewrite Eqn. 2.13 as

$$
\left.\sqrt{\left|E_{\Pi}\right|}\left|E_{\Pi}\right\rangle=\sum_{\Pi^{\prime} \vdash 2 k} \zeta\left(\Pi, \Pi^{\prime}\right) \sqrt{\left|I_{\Pi^{\prime}}\right|} I_{\Pi^{\prime}}\right\rangle,
$$

then we can use $\mu$ to express $\left|I_{\Pi}\right\rangle$ in terms of the $\left|E_{\Pi}\right\rangle$ as

$$
\sqrt{\left|I_{\Pi}\right|}\left|I_{\Pi}\right\rangle=\sum_{\Pi^{\prime} \vdash 2 k} \mu\left(\Pi, \Pi^{\prime}\right) \sqrt{\left|E_{\Pi^{\prime}}\right|}\left|E_{\Pi^{\prime}}\right\rangle .
$$

This approach is a generalisation of inclusion-exclusion known as Möbius inversion, and the function $\mu$ is called the Möbius function (see Chapter 3 of [13] for more background). For the case of the refinement partial order, the Möbius function is known:

Lemma 2.4 ([11], Section 7).

$$
\mu\left(\Pi, \Pi^{\prime}\right)=(-1)^{|\Pi|-\left|\Pi^{\prime}\right|} \prod_{i=1}^{\left|\Pi^{\prime}\right|}\left(b_{i}-1\right) !
$$

where $b_{i}$ is the number of blocks of $\Pi$ in the $i^{\text {th }}$ block of $\Pi^{\prime}$.

We can use this to evaluate sums involving the Möbius function for the refinement order.

Lemma 2.5.

$$
\sum_{\Pi^{\prime} \geq \Pi}\left|\mu\left(\Pi, \Pi^{\prime}\right)\right| x^{\left|\Pi^{\prime}\right|}=x^{(|\Pi|)}
$$

where $x$ is arbitrary and $x^{(n)}$ is the rising factorial $x(x+1) \cdots(x+n-1)$.

Proof. Start with $\left|\mu\left(\Pi, \Pi^{\prime}\right)\right|=(-1)^{|\Pi|-\left|\Pi^{\prime}\right|} \mu\left(\Pi, \Pi^{\prime}\right)$ to obtain

$$
\begin{aligned}
\sum_{\Pi^{\prime} \geq \Pi}\left|\mu\left(\Pi, \Pi^{\prime}\right)\right| x^{\left|\Pi^{\prime}\right|} & =(-1)^{|\Pi|} \sum_{\Pi^{\prime} \geq \Pi} \mu\left(\Pi, \Pi^{\prime}\right)(-x)^{\left|\Pi^{\prime}\right|} & & \\
& =(-1)^{|\Pi|} \sum_{\Pi^{\prime} \geq \Pi} \mu\left(\Pi, \Pi^{\prime}\right) \sum_{\Pi^{\prime \prime} \geq \Pi^{\prime}} \zeta\left(\Pi^{\prime}, \Pi^{\prime \prime}\right)(-x)_{\left(\left|\Pi^{\prime \prime}\right|\right)} & & \text { using Eqn. 2.12 } \\
& =(-1)^{|\Pi|}(-x)_{(|\Pi|)} & & \text { by Möbius inversion } \\
& =x^{(|\Pi|)} & & \text { using }(-x)_{(n)}=(-1)^{n} x^{(n)} .
\end{aligned}
$$


We will mostly be interested in the special case $x=1$ :

Corollary 2.6.

$$
\sum_{\Pi^{\prime} \geq \Pi}\left|\mu\left(\Pi, \Pi^{\prime}\right)\right|=|\Pi| !
$$

Using $\left|\mu\left(\Pi, \Pi^{\prime}\right)\right| \geq 1$ and the fact that $\Pi \geq\{\{1\}, \ldots,\{n\}\}$ for all $\Pi \vdash n$, we obtain a bound on the total number of partitions.

Corollary 2.7. The Bell numbers $\beta_{n}$ satisfy $\beta_{n} \leq n$ !.

\subsection{Fixed Points of a Quantum Expander}

We now turn to $V_{\mathcal{U}(N)}$, the space fixed by the quantum expander. By Schur-Weyl duality (see e.g. [4]), the only operators on $\left(\mathbb{C}^{N}\right)^{\otimes k}$ to commute with all $U^{\otimes k}$ are linear combinations of subsystem permutations

$$
S(\pi)=\sum_{n_{1}=1}^{N} \cdots \sum_{n_{k}=1}^{N}\left|n_{\pi^{-1}(1)}, \ldots n_{\pi^{-1}(k)}\right\rangle\left\langle n_{1}, \ldots, n_{k}\right|
$$

for $\pi \in \mathcal{S}_{k}$. The equivalent statement for $V_{\mathcal{U}(N)}$ is that the only states invariant under all $U^{\otimes k, k}$ are of the form

$$
\frac{1}{\sqrt{N^{k}}} \sum_{n_{1}, \ldots, n_{k} \in[N]}\left|n_{1}, \ldots, n_{k}, n_{\pi(1)}, \ldots, n_{\pi(k)}\right\rangle,
$$

for some permutation $\pi \in \mathcal{S}_{k}$. Since $\mathcal{E}_{\mathcal{U}(N)}^{k}=\mathbb{E}\left[U^{\otimes k, k}\right]$ projects onto the set of states that is invariant under all $U^{\otimes k, k}$, it follows that $V_{\mathcal{U}(N)}$ is equal to the span of the states in Eqn. 2.19. Now we relate these states to our previous notation.

Definition 2.8. For $\pi \in \mathcal{S}_{k}$, define the partition corresponding to $\pi$ by

$$
P(\pi)=\{\{1, k+\pi(1)\},\{2, k+\pi(2)\}, \ldots,\{k, k+\pi(k)\}\} .
$$

Then the state in Eqn. 2.19 is simply $\left|E_{P(\pi)}\right\rangle$, and so

$$
V_{\mathcal{U}(N)}=\operatorname{span}\left\{\left|E_{P(\pi)}\right\rangle: \pi \in \mathcal{S}_{k}\right\} .
$$

Note that the classical expander has many more fixed points than just the $\left|E_{P(\pi)}\right\rangle$. The main task in constructing a quantum expander from a classical one is to modify the classical expander to decay the fixed points that should not be fixed by the quantum expander.

\subsection{Fourier Transform in the Matrix Element Basis}

Since we make use of the Fourier transform, we will need to know how it acts on a matrix element. We find

$$
\begin{aligned}
\mathcal{F}^{\otimes k, k}|\mathbf{m}\rangle & =\frac{1}{N^{k}} \sum_{\mathbf{n}} \exp \left(\frac{2 \pi i}{N}\left(m_{1} n_{1}+\ldots m_{k} n_{k}-m_{k+1} n_{k+1}-\ldots-m_{2 k} n_{2 k}\right)\right)|\mathbf{n}\rangle \\
& =\frac{1}{N^{k}} \sum_{\mathbf{n}} \omega^{\mathbf{m} \cdot \mathbf{n}}|\mathbf{n}\rangle
\end{aligned}
$$


where

$$
\mathbf{m} . \mathbf{n}=m_{1} n_{1}+\ldots+m_{k} n_{k}-m_{k+1} n_{k+1}-\ldots-m_{2 k} n_{2 k}
$$

We will also find it convenient to estimate the matrix elements $\left\langle E_{\Pi_{1}}\left|\mathcal{F}^{\otimes k, k}\right| E_{\Pi_{2}}\right\rangle$. The properties we require are proven in the following lemmas.

Lemma 2.9. Choose any $\Pi_{1}, \Pi_{2} \vdash 2 k$. Let $\mathbf{m} \in \Pi_{1}$ and $\mathbf{n} \in \Pi_{2}$. Call the free indices of $\mathbf{m} \tilde{m}_{i}$ for $1 \leq i \leq\left|\Pi_{1}\right|$. Then let $\mathbf{m} . \mathbf{n}=\sum_{i=1}^{\left|\Pi_{1}\right|} \sum_{j=1}^{2 k} \tilde{m}_{i} A_{i, j} n_{j}$ where $A_{i, j}$ is a $\left|\Pi_{1}\right| \times 2 k$ matrix with entries in $\{0,1,-1\}$ which depends on $\Pi_{1}$ (but not $\left.\Pi_{2}\right)$. Then

$$
\left\langle E_{\Pi_{1}}\left|\mathcal{F}^{\otimes k, k}\right| E_{\Pi_{2}}\right\rangle=N^{-k+\frac{\left|\Pi_{1}\right|-\left|\Pi_{2}\right|}{2}} \sum_{\mathbf{n} \in E_{\Pi_{2}}} \mathbb{I}\left(\sum_{j} A_{i, j} n_{j} \equiv 0 \bmod N \forall i\right)
$$

where $\mathbb{I}$ is the indicator function.

Proof. Simply perform the $\mathbf{m}$ sum in

$$
\left\langle E_{\Pi_{1}}\left|\mathcal{F}^{\otimes k, k}\right| E_{\Pi_{2}}\right\rangle=N^{-\left(k+\frac{\left|\Pi_{1}\right|+\left|\Pi_{2}\right|}{2}\right)} \sum_{\mathbf{m} \in E_{\Pi_{1}}} \sum_{\mathbf{n} \in E_{\Pi_{2}}} \omega^{\mathbf{m} \cdot \mathbf{n}}
$$

Lemma 2.10. $\left\langle E_{\Pi_{1}}\left|\mathcal{F}^{\otimes k, k}\right| E_{\Pi_{2}}\right\rangle$ is real and positive.

Proof. Since all entries in the sum in Eqn. 2.22 are nonnegative and at least one $(\mathbf{n}=0)$ is strictly positive, Lemma 2.9 implies the result.

Lemma 2.11. If $\Pi_{1}^{\prime} \leq \Pi_{1}$ and $\Pi_{2}^{\prime} \leq \Pi_{2}$ then

$$
\sqrt{\left|E_{\Pi_{1}}\right| \cdot\left|E_{\Pi_{2}}\right|}\left\langle E_{\Pi_{1}}\left|\mathcal{F}^{\otimes k, k}\right| E_{\Pi_{2}}\right\rangle \leq \sqrt{\left|E_{\Pi_{1}^{\prime}}\right| \cdot \mid E_{\Pi_{2}^{\prime}}}\left\langle\left\langle E_{\Pi_{1}^{\prime}}\left|\mathcal{F}^{\otimes k, k}\right| E_{\Pi_{2}^{\prime}}\right\rangle\right.
$$

Proof. We prove first the special case when $\Pi_{1}^{\prime}=\Pi_{1}$, but $\Pi_{2}^{\prime} \leq \Pi_{2}$ is arbitrary. Recall that $\Pi_{2}^{\prime} \leq \Pi_{2}$ implies that $E_{\Pi_{2}} \subseteq E_{\Pi_{2}^{\prime}}$. Now the LHS of Eqn. 2.24 equals

$$
\begin{aligned}
N^{-k} \sum_{\mathbf{m} \in E_{\Pi_{1}}, \mathbf{n} \in E_{\Pi_{2}}} \exp \left(\frac{2 \pi i}{N} \mathbf{m} \cdot \mathbf{n}\right) & =N^{\left|\Pi_{1}\right|-k} \sum_{\mathbf{n} \in E_{\Pi_{2}}} \mathbb{I}\left(\sum_{j} A_{i, j} n_{j} \equiv 0 \bmod N \forall i\right) \\
& =N^{\left|\Pi_{1}\right|-k} \sum_{\mathbf{n} \in E_{\Pi_{2}^{\prime}}} \mathbb{I}\left(\mathbf{n} \in E_{\Pi_{2}}\right) \mathbb{I}\left(\sum_{j} A_{i, j} n_{j} \equiv 0 \bmod N \forall i\right) \\
& \leq N^{\left|\Pi_{1}\right|-k} \sum_{\mathbf{n} \in E_{\Pi_{2}^{\prime}}} \mathbb{I}\left(\sum_{j} A_{i, j} n_{j} \equiv 0 \bmod N \forall i\right) \\
& =\sqrt{\left|E_{\Pi_{1}}\right|\left|E_{\Pi_{2}^{\prime}}\right|\left\langle E_{\Pi_{1}}\right| \mathcal{F}} \otimes k, k\left|E_{\Pi_{2}^{\prime}}\right\rangle,
\end{aligned}
$$

as desired. To prove Eqn. 2.24 we repeat this argument, interchanging the roles of $\Pi_{1}$ and $\Pi_{2}$ and use the fact that $\left\langle E_{\Pi_{1}}\left|\mathcal{F}^{\otimes k, k}\right| E_{\Pi_{2}}\right\rangle$ is symmetric in $\Pi_{1}$ and $\Pi_{2}$.

\section{Lemma 2.12.}




$$
\left\langle E_{\Pi_{1}}\left|\mathcal{F}^{\otimes k, k}\right| E_{\Pi_{2}}\right\rangle \leq N^{-\frac{1}{2}\left|2 k-\left(\left|\Pi_{1}\right|+\left|\Pi_{2}\right|\right)\right|}
$$

Proof. Here, there are two cases to consider. The simpler case is when $\left|\Pi_{1}\right|+\left|\Pi_{2}\right| \leq 2 k$. Here we simply apply the inequality

$$
\sum_{\mathbf{m} \in E_{\Pi_{1}}, \mathbf{n} \in E_{\Pi_{2}}} \exp \left(\frac{2 \pi i}{N} \mathbf{m} . \mathbf{n}\right) \leq\left|E_{\Pi_{1}}\right|\left|E_{\Pi_{2}}\right|=N^{\left|\Pi_{1}\right|\left|\Pi_{2}\right|}
$$

to Eqn. 2.23, and conclude that $\left\langle E_{\Pi_{1}}\left|\mathcal{F}^{\otimes k, k}\right| E_{\Pi_{2}}\right\rangle \leq N^{\frac{\left|\Pi_{1}\right|+\left|\Pi_{2}\right|}{2}-k}$.

Next, we would like to prove that

$$
\left\langle E_{\Pi_{1}}\left|\mathcal{F}^{\otimes k, k}\right| E_{\Pi_{2}}\right\rangle \leq N^{k-\frac{\left|\Pi_{1}\right|+\left|\Pi_{2}\right|}{2}} .
$$

Here we use Lemma 2.11 with $\Pi_{1}^{\prime}=\Pi_{1}$ and $\Pi_{2}^{\prime}=\{\{1\},\{2\}, \ldots,\{2 k\}\}$, the maximally refined partition. Note that $\left|E_{\Pi_{2}^{\prime}}\right|=N^{2 k}$ and $\mathcal{F}^{\otimes k, k}\left|E_{\Pi_{2}^{\prime}}\right\rangle=|0\rangle$. Thus

$$
\left\langle E_{\Pi_{1}}\left|\mathcal{F}^{\otimes k, k}\right| E_{\Pi_{2}}\right\rangle \leq N^{k-\frac{\left|\Pi_{2}\right|}{2}}\left\langle E_{\Pi_{1}}\left|\mathcal{F}^{\otimes k, k}\right| E_{\Pi_{2}^{\prime}}\right\rangle=N^{k-\frac{\left|\Pi_{2}\right|}{2}}\left\langle E_{\Pi_{1}} \mid 0\right\rangle=N^{k-\frac{\left|\Pi_{1}\right|+\left|\Pi_{2}\right|}{2}},
$$

establishing Eqn. 2.26,

\section{Lemma 2.13.}

If $\Pi_{1}=\Pi_{2}=P(\pi)$ then $\left\langle E_{\Pi_{1}}\left|\mathcal{F}^{\otimes k, k}\right| E_{\Pi_{2}}\right\rangle=1$. If, for any $\Pi_{1}, \Pi_{2}$ with $\left|\Pi_{1}\right|+\left|\Pi_{2}\right|=2 k$, either condition isn't met (i.e. either $\Pi_{1} \neq \Pi_{2}$ or there does not exist $\pi \in \mathcal{S}_{k}$ such that $P(\pi)=\Pi_{1}=\Pi_{2}$ ) then

$$
\left\langle E_{\Pi_{1}}\left|\mathcal{F}^{\otimes k, k}\right| E_{\Pi_{2}}\right\rangle \leq \frac{2^{k}}{N}
$$

for $N>k$.

Proof. In Lemma 2.14, we introduce the $\Pi_{1} \times \Pi_{2}$ matrix $\tilde{A}$ with the property that

$$
\mathbf{m} . \mathbf{n}=\sum_{i=1}^{\left|\Pi_{1}\right|} \sum_{j=1}^{\left|\Pi_{2}\right|} \tilde{m}_{i} \tilde{A}_{i, j} \tilde{n}_{j}
$$

for all $\mathbf{m} \in \Pi_{1}$ and $\mathbf{n} \in \Pi_{2}$ where $\tilde{m}_{j}$ and $\tilde{n}_{j}$ are the free indices of $\mathbf{m}$ and $\mathbf{n}$. This is similar to the matrix $A$ introduced in Lemma 2.9 except only the free indices of $\mathbf{n}$ are considered.

For $\Pi_{1}=\Pi_{2}=P(\pi)$, Lemma 2.14 implies that $\tilde{A}=0$, or equivalently $\mathbf{m} . \mathbf{n}=0$ for all $\mathbf{m}, \mathbf{n} \in P(\pi)$. Using $\left|\Pi_{1}\right|+\left|\Pi_{2}\right|=2 k,\left\langle E_{\Pi_{1}}\left|\mathcal{F}^{\otimes k, k}\right| E_{\Pi_{2}}\right\rangle=1$.

Otherwise we have $\left(\Pi_{1}, \Pi_{2}\right) \notin\left\{(P(\pi), P(\pi)): \pi \in \mathcal{S}_{k}\right\}$ with $\left|\Pi_{1}\right|+\left|\Pi_{2}\right|=2 k$. For all these, Lemma 2.14 implies that $\tilde{A}$ is nonzero (for $N>k$, no entries in $\tilde{A}$ can be $>N$ or $<-N$ so $\tilde{A} \equiv 0 \bmod N$ is equivalent to $\tilde{A}=0)$. Fix an $i$ for which the $i^{\text {th }}$ row of $\tilde{A}$ is nonzero. We wish to count the number of $\left(\tilde{n}_{1}, \ldots, \tilde{n}_{\mid \Pi_{2}}\right)$ such that $\sum_{j} \tilde{A}_{i, j} \tilde{n}_{j} \equiv 0 \bmod N$. Assume that each $\tilde{A}_{i, j}$ divides $N$ and is nonnegative; if not, we can replace $\tilde{A}_{i, j}$ with $\operatorname{GCD}\left(\left|\tilde{A}_{i, j}\right|, N\right)$ by a suitable change of variable for $\tilde{n}_{j}$. Let $L$ denote the least common multiple of the nonzero $\tilde{A}_{i, j}$. Note that $L$ divides $N$. We claim that if $\tilde{\mathbf{n}}$ is chosen randomly from $[N]^{\left|\Pi_{2}\right|}$ then the probability that $\sum_{j} \tilde{A}_{i, j} \tilde{n}_{j} \equiv 0 \bmod N$ is $\leq L / N$. Equivalently, there are $\leq L N^{\left|\Pi_{2}\right|-1}$ choices of $\tilde{\mathbf{n}}$ such that $\sum_{j} \tilde{A}_{i, j} \tilde{n}_{j} \equiv 0 \bmod N$. To prove this claim, write $\tilde{n}_{j}$ as

$$
\tilde{n}_{j}=\frac{L}{A_{i, j}} x_{j}+y_{j}
$$


for some $0 \leq x_{j}<A_{i, j} N / L$ and $0 \leq y_{j}<L / A_{i, j}$. Randomly choosing the $\tilde{n}_{j}$ is equivalent to randomly choosing $x_{1}, \ldots, x_{\left|\Pi_{2}\right|}$ and $y_{1}, \ldots, y_{\left|\Pi_{2}\right|}$ according to the above ranges. The condition

$$
0 \equiv \sum_{j} \tilde{A}_{i, j} \tilde{n}_{j} \equiv L \sum_{j} x_{j}+\sum_{j} \tilde{A}_{i, j} y_{j} \bmod N
$$

is equivalent to demanding that $\sum_{j} \tilde{A}_{i, j} y_{j} \equiv 0 \bmod L$ and $\sum_{j} x_{j} \equiv C \bmod N / L$, for some $C$ depending on the $y_{j}$. If we relax the condition on the $y_{j}$, then there is still only a $L / N$ probability that $\sum_{j} x_{j} \equiv C \bmod N / L$. Thus

$$
\sum_{\tilde{n}_{1}, \ldots, \tilde{n}_{\left|\Pi_{2}\right|} \in[N]} \mathbb{I}\left(\sum_{j} \tilde{A}_{i, j} \tilde{n}_{j} \equiv 0 \bmod N \forall i=1, \ldots,\left|\Pi_{1}\right|\right) \leq L N^{\left|\Pi_{2}\right|-1} \leq 2^{k} N^{\left|\Pi_{2}\right|-1},
$$

where this last inequality is because $L \leq \prod_{j=1}^{\left|\Pi_{2}\right|}\left|\tilde{A}_{i, j}\right|$ and $\sum_{j=1}^{\left|\Pi_{2}\right|}\left|\tilde{A}_{i, j}\right| \leq 2 k$. Substituting Eqn. 2.31 into Eqn. 2.22 (which we can trivially modify to apply for $\tilde{A}$ rather than just $A$ ), we find that

$$
\left\langle E_{\Pi_{1}}\left|\mathcal{F}^{\otimes k, k}\right| E_{\Pi_{2}}\right\rangle \leq \frac{2^{k}}{N} N^{-k+\frac{\left|\Pi_{1}\right|+\left|\Pi_{2}\right|}{2}}=\frac{2^{k}}{N},
$$

thus establishing Eqn. 2.27.

Lemma 2.14. Let $\tilde{A}$ be the matrix such that $\mathbf{m . n}=\sum_{i=1}^{\left|\Pi_{1}\right|} \sum_{j=1}^{\left|\Pi_{2}\right|} \tilde{m}_{i} \tilde{A}_{i, j} \tilde{n}_{j}$ for all $\mathbf{m} \in \Pi_{1}$ and $\mathbf{n} \in \Pi_{2}$ where $\tilde{m}_{j}$ and $\tilde{n}_{j}$ are the free indices of $\mathbf{m}$ and $\mathbf{n}$. Then $\tilde{A}=0$ if and only if $\Pi_{1}=\Pi_{2} \geq P(\pi)$ for some $\pi \in \mathcal{S}_{k}$

Proof. We first consider $\Pi_{1}=\Pi_{2}=P(\pi)$ for the "if" direction. Note that for any $\mathbf{m}, \mathbf{n} \in E_{P(\pi)}$, we have

$$
\mathbf{m . n}=\sum_{j=1}^{k} m_{j} n_{j}-\sum_{j=1}^{k} m_{\pi(j)} n_{\pi(j)}=0 .
$$

This implies that $\tilde{A}=0$. Now, choose any $\Pi_{1} \geq P(\pi)$ and $\Pi_{2} \geq P(\pi)$. Then for any $\mathbf{m} \in \Pi_{1}$ and $\mathbf{n} \in \Pi_{2}, \mathbf{m}, \mathbf{n} \in P(\pi)$. This means Eqn. 2.32 holds for this case so $\tilde{A}=0$ also.

On the other hand, suppose that $\tilde{A}=0$. We will argue that this implies the existence of a permutation $\pi$ such that $\Pi_{1}, \Pi_{2} \geq P(\pi)$, thus establishing the "only if" direction.

Let $\Pi_{1, j}\left(\right.$ resp. $\left.\Pi_{2, j}\right)$ denote the $j^{\text {th }}$ block of $\Pi_{1}\left(\right.$ resp. $\left.\Pi_{2}\right)$. Then

$$
\tilde{A}_{i, j}=\sum_{\substack{i^{\prime} \in \Pi_{1, i} \\ j^{\prime} \in \Pi_{2, j}}} \Lambda_{i^{\prime}, j^{\prime}}
$$

where $\Lambda_{i^{\prime}, j^{\prime}}$ is defined to be

$$
\Lambda_{i^{\prime}, j^{\prime}}= \begin{cases}1 & \text { if } i^{\prime}=j^{\prime} \in\{1, \ldots, k\} \\ -1 & \text { if } i^{\prime}=j^{\prime} \in\{k+1, \ldots, 2 k\} \\ 0 & \text { if } i^{\prime} \neq j^{\prime}\end{cases}
$$

If $\tilde{A}=0$ then for each $i, j$ we have

$$
\left|\Pi_{1, i} \cap \Pi_{2, j} \cap\{1, \ldots, k\}\right|=\left|\Pi_{1, i} \cap \Pi_{2, j} \cap\{k+1, \ldots, 2 k\}\right| .
$$


Denote the meet of $\Pi_{1}$ and $\Pi_{2}, \Pi_{1} \wedge \Pi_{2}$ to be the greatest lower bound of $\Pi_{1}$ and $\Pi_{2}$, or equivalently the unique partition with the fewest blocks that satisfies $\Pi_{1} \wedge \Pi_{2} \leq \Pi_{1}$ and $\Pi_{1} \wedge \Pi_{2} \leq$ $\Pi_{2}$. The blocks of $\Pi_{1} \wedge \Pi_{2}$ are simply all of the nonempty sets $\Pi_{1, i} \cap \Pi_{2, j}$, for $i=1, \ldots,\left|\Pi_{1}\right|$ and $j=1, \ldots,\left|\Pi_{2}\right|$. Thus, Eqn. 2.33 implies that each block of $\Pi_{1} \wedge \Pi_{2}$ contains an equal number of indices from $\{1, \ldots, k\}$ as it does from $\{k+1, \ldots, 2 k\}$. This implies the existence of a permutation $\pi \in \mathcal{S}_{k}$ such that $\{i, k+\pi(i)\}$ is contained in a single block of $\Pi_{1} \wedge \Pi_{2}$ for each $i=1, \ldots, k$. Equivalently $\Pi_{1} \wedge \Pi_{2} \geq P(\pi)$, implying that $\Pi_{1} \geq P(\pi)$ and $\Pi_{2} \geq P(\pi)$.

\subsection{Proof of Lemma 2.2}

Proof. We would like to show that, for any unit vector $|\psi\rangle \in V_{0},\left|\left\langle\psi\left|\mathcal{F}^{\otimes k, k}\right| \psi\right\rangle\right|^{2} \leq 2(2 k)^{4 k} / \sqrt{N}$. Our strategy will be to calculate the matrix elements of $\mathcal{F}^{\otimes k, k}$ in the $\left|I_{\Pi}\right\rangle$ and $\left|E_{\pi}\right\rangle$ bases. While the $\left|I_{\Pi}\right\rangle$ states are orthonormal, we will see that the $\left\langle E_{\Pi_{1}}\left|\mathcal{F}^{\otimes k, k}\right| E_{\Pi_{2}}\right\rangle$ matrix elements are easier to calculate. We then use Möbius functions to express $\left|I_{\Pi}\right\rangle$ in terms of $\left|E_{\Pi}\right\rangle$.

Consider the matrix $\mathcal{E}_{\mathcal{S}_{N}}^{2 k} \mathcal{F}^{\otimes k, k} \mathcal{E}_{\mathcal{S}_{N}}^{2 k}$. It has $k$ ! unit eigenvalues, corresponding to the $k$ !-dimensional space $V_{\mathcal{U}(N)}$. Call the $k !+1^{\text {st }}$ largest eigenvalue $\lambda_{A}$. We bound $\lambda_{A}$ with

$$
\begin{aligned}
k !+\lambda_{A}^{2} & \leq \operatorname{tr}\left(\mathcal{E}_{\mathcal{S}_{N}}^{2 k} \mathcal{F}^{\otimes k, k} \mathcal{E}_{\mathcal{S}_{N}}^{2 k}\right)^{2} \\
& =\sum_{\Pi_{1}, \Pi_{2} \vdash 2 k}\left|\left\langle I_{\Pi_{1}}\left|\mathcal{F}^{\otimes k, k}\right| I_{\Pi_{2}}\right\rangle\right|^{2} .
\end{aligned}
$$

We divide the terms in Eqn. 2.34 into four types.

1. The leading-order contribution comes from the $k$ ! terms of the form $\Pi_{1}=\Pi_{2}=P(\pi)$ for $\pi \in \mathcal{S}_{k}$. We bound them with the trivial upper bound

$$
\left|\left\langle I_{\Pi_{1}}\left|\mathcal{F}^{\otimes k, k}\right| I_{\Pi_{2}}\right\rangle\right|^{2} \leq 1
$$

(which turns out to be nearly tight). We will then show that the remaining terms are all $k^{O(k)} / N$.

2. If $\left|\Pi_{1}\right|+\left|\Pi_{2}\right|<2 k$ then

$$
\begin{aligned}
\left|\left\langle I_{\Pi_{1}}\left|\mathcal{F}^{\otimes k, k}\right| I_{\Pi_{2}}\right\rangle\right|^{2} & =\frac{1}{\left|I_{\Pi_{1}}\right| \cdot\left|I_{\Pi_{2}}\right| N^{2 k}}\left|\sum_{\substack{\mathbf{m} \in \Pi_{1} \\
\mathbf{n} \in \Pi_{2}}} e^{\frac{2 \pi i \mathbf{m} . \mathbf{n}}{N}}\right|^{2} \\
& \leq \frac{\left|I_{\Pi_{1}}\right| \cdot\left|I_{\Pi_{2}}\right|}{N^{2 k}} \\
& \leq N^{\left|\Pi_{1}\right|+\left|\Pi_{2}\right|-2 k} \leq \frac{1}{N}
\end{aligned}
$$

where in the last line we have used the fact that $\left|I_{\Pi}\right| \leq\left|E_{\Pi}\right|=N^{|\Pi|}$.

3. If $\left|\Pi_{1}\right|+\left|\Pi_{2}\right|>2 k$ then we will show that

$$
\left|\left\langle I_{\Pi_{1}}\left|\mathcal{F}^{\otimes k, k}\right| I_{\Pi_{2}}\right\rangle\right|^{2} \leq \frac{4 \cdot(2 k !)^{2}}{N}
$$


4. If $\left|\Pi_{1}\right|+\left|\Pi_{2}\right|=2 k$ but either $\Pi_{1} \neq \Pi_{2}$ or there is no $\pi \in \mathcal{S}_{k}$ satisfying $P(\pi)=\Pi_{1}=\Pi_{2}$, then we will show that

$$
\left|\left\langle I_{\Pi_{1}}\left|\mathcal{F}^{\otimes k, k}\right| I_{\Pi_{2}}\right\rangle\right|^{2} \leq \frac{\left((2 k) !+2^{k}\right)^{2}}{N^{2}} \leq \frac{4 \cdot(2 k !)^{2}}{N}
$$

To establish these last two claims, we will find it useful to express $\left|I_{\Pi}\right\rangle$ in terms of the various $\left|E_{\Pi}\right\rangle$ states.

Lemmas 2.12 and 2.13 can now be used together with the Möbius function to bound $\left|\left\langle I_{\Pi_{1}}\left|\mathcal{F}{ }^{\otimes k, k}\right| I_{\Pi_{2}}\right\rangle\right|^{2}$. First, suppose $\left|\Pi_{1}\right|+\left|\Pi_{2}\right|>2 k$. Then

$$
\begin{aligned}
\left|\left\langle I_{\Pi_{1}}\left|\mathcal{F}^{\otimes k, k}\right| I_{\Pi_{2}}\right\rangle\right| & =\left|\sum_{\substack{\Pi_{1}^{\prime} \geq \Pi_{1} \\
\Pi_{2}^{\prime} \geq \Pi_{2}}} \sqrt{\frac{\left|E_{\Pi_{1}^{\prime}}\right|\left|E_{\Pi_{2}^{\prime}}\right|}{\left|I_{\Pi_{1}}\right|\left|I_{\Pi_{2}}\right|}} \mu\left(\Pi_{1}, \Pi_{1}^{\prime}\right) \mu\left(\Pi_{2}, \Pi_{2}^{\prime}\right)\left\langle E_{\Pi_{1}}\left|\mathcal{F}^{\otimes k, k}\right| E_{\Pi_{2}}\right\rangle\right| \\
& \leq \sum_{\substack{\Pi_{1}^{\prime} \geq \Pi_{1} \\
\Pi_{2}^{\prime} \geq \Pi_{2}}} \sqrt{\frac{\left|E_{\Pi_{1}^{\prime}}\right|\left|E_{\Pi_{2}^{\prime}}\right|}{\left|I_{\Pi_{1}}\right|\left|I_{\Pi_{2}}\right|}}\left|\mu\left(\Pi_{1}, \Pi_{1}^{\prime}\right) \mu\left(\Pi_{2}, \Pi_{2}^{\prime}\right)\right|\left\langle E_{\Pi_{1}^{\prime}}\left|\mathcal{F}^{\otimes k, k}\right| E_{\Pi_{2}^{\prime}}\right\rangle \\
& \leq \frac{N^{k}}{\sqrt{\left|I_{\Pi_{1}}\right|\left|I_{\Pi_{2}}\right|}} \sum_{\Pi_{1}^{\prime} \geq \Pi_{1}}\left|\mu\left(\Pi_{1}^{\prime}, \Pi_{1}\right) \mu\left(\Pi_{2}^{\prime}, \Pi_{2}\right)\right| \\
& =\frac{N^{k}\left|\Pi_{1}\right| !\left|\Pi_{2}\right| !}{\sqrt{(N)})_{\left|\Pi_{1}\right|}(N)_{\mid \Pi_{2}} \mid} \\
& \leq \frac{2 \cdot(2 k) !}{\sqrt{N}}
\end{aligned}
$$

by Lemma 2.12

by Corollary 2.6

In the last step, we have assumed that $4 k^{2}<N$, so that $(N)_{\ell} \geq N^{\ell} / 2$ for any $\ell \leq 2 k$. We have also made use of the fact that (still assuming $4 k^{2}<N$ ) Eqn. 2.36 is maximised when $\left|\Pi_{1}\right|+\left|\Pi_{2}\right|=2 k+1$, and in particular, when one of $\left|\Pi_{1}\right|,\left|\Pi_{2}\right|$ is equal to $2 k$ and the other is equal to 1.

A similar analysis applies to the pairs $\Pi_{1}, \Pi_{2}$ with $\left|\Pi_{1}\right|+\left|\Pi_{2}\right|=2 k$, but with $\left(\Pi_{1}, \Pi_{2}\right) \notin$ $\left\{(P(\pi), P(\pi)): \pi \in \mathcal{S}_{k}\right\}$. In this case,

$$
\begin{aligned}
&\left\langle I_{\Pi_{1}}\left|\mathcal{F}^{\otimes k, k}\right| I_{\Pi_{2}}\right\rangle= \sqrt{\frac{\left|E_{\Pi_{1}}\right|\left|E_{\Pi_{2}}\right|}{\left|I_{\Pi_{1}}\right|\left|I_{\Pi_{2}}\right|}}\left\langle E_{\Pi_{1}}\left|\mathcal{F}^{\otimes k, k}\right| E_{\Pi_{2}}\right\rangle+ \\
& \sum_{\substack{\Pi_{1}^{\prime} \geq \Pi_{1}, \Pi_{2}^{\prime} \geq \Pi_{2} \\
\left(\Pi_{1}^{\prime}, \Pi_{2}^{\prime}\right) \neq\left(\Pi_{1}, \Pi_{2}\right)}} \sqrt{\frac{\left|E_{\Pi_{1}^{\prime}}\right|\left|E_{\Pi_{2}^{\prime}}\right|}{\left|I_{\Pi_{1}}\right|\left|I_{\Pi_{2}}\right|}} \mu\left(\Pi_{1}, \Pi_{1}^{\prime}\right) \mu\left(\Pi_{2}, \Pi_{2}^{\prime}\right)\left\langle E_{\Pi_{1}^{\prime}}\left|\mathcal{F}^{\otimes k, k}\right| E_{\Pi_{2}^{\prime}}\right\rangle
\end{aligned}
$$

We now use Lemmas 2.13 and 2.12 to bound each of the two terms. For the first term, we use Eqn. 2.27 to upper bound it with $2^{k} / N$. For each choice of $\Pi_{1}^{\prime}$ and $\Pi_{2}^{\prime}$ in the second sum, we have $\left|\Pi_{1}^{\prime}\right|+\left|\Pi_{2}^{\prime}\right| \leq 2 k-1$. Thus we can upper bound the absolute value of the second term in Eqn. 2.37 with

$$
\frac{1}{\sqrt{\left|I_{\Pi_{1}}\right|\left|I_{\Pi_{2}}\right|}} \sum_{\substack{\Pi_{1}^{\prime} \geq \Pi_{1}, \Pi_{2}^{\prime} \geq \Pi_{2} \\\left(\Pi_{1}^{\prime}, \Pi_{2}^{\prime}\right) \neq\left(\Pi_{1}, \Pi_{2}\right)}}\left|\mu\left(\Pi_{1}, \Pi_{1}^{\prime}\right) \mu\left(\Pi_{2}, \Pi_{2}^{\prime}\right)\right| N^{\left|\Pi_{1}^{\prime}\right|+\left|\Pi_{2}^{\prime}\right|-k} \leq \frac{2 \cdot\left|\Pi_{1}\right| ! \cdot\left|\Pi_{2}\right| !}{N} \leq \frac{(2 k) !}{N} .
$$


We combine the two terms and square to establish Eqn. 2.35d,

We now put together the components from Eqn. 2.35 to upper bound Eqn. 2.34, and find that

$$
k !+\lambda_{A}^{2} \leq k !+\beta_{2 k}^{2} \frac{4 \cdot(2 k !)^{2}}{N},
$$

implying that $\lambda_{A} \leq 2 \beta_{2 k}(2 k !) / \sqrt{N} \leq 2(2 k)^{4 k} / \sqrt{N}$. This concludes the proof of Lemma 2.2 .

\section{Conclusions}

We have shown how efficient quantum tensor product expanders can be constructed from efficient classical tensor product expanders. We show how this can be used to give an efficient construction of unitary $k$ designs for any $k$. Unfortunately our results do not work for all dimensions; we require the dimension $N$ to be $\Omega\left((2 k)^{8 k}\right)$. While tighter analysis of our construction could likely improve this, our construction does not work for $N<2 k$. Constructions of expanders for all dimensions remains an open problem.

\section{Acknowledgments}

We are grateful for funding from the Army Research Office under grant W9111NF-05-1-0294, the European Commission under Marie Curie grants ASTQIT (FP6-022194) and QAP (IST-200515848), and the U.K. Engineering and Physical Science Research Council through "QIP IRC." RL would like to thank Markus Grassl and Andreas Winter for helpful discussions. RL is also extremely grateful to Andreas Winter and the rest of the Centre for Quantum Technologies, National University of Singapore, where part of this research was carried out, for their kind hospitality.

\section{References}

[1] A. Ambainis, J. Bouda, and A. Winter. Tamper-resistant encryption of quantum information, 2008. arXiv:0808.0353.

[2] A. Ambainis and E. Emerson. Quantum t-designs: t-wise Independence in the Quantum World. Computational Complexity 2007, 2007. arXiv:quant-ph/0701126v2.

[3] A. Ambainis and A. Smith. Small Pseudo-Random Families of Matrices: Derandomizing Approximate Quantum Encryption. Lecture Notes in Computer Science, 3122/2004:249260, 2004. arXiv:quant-ph/0404075.

[4] R. Goodman and N. Wallach. Representations and Invariants of the Classical Groups. Cambridge University Press, Cambridge, UK, 1998.

[5] A. W. Harrow and R. A. Low. Random quantum circuits are approximate 2-designs, 2008. arXiv:0802.1919.

[6] M. B. Hastings and A. W. Harrow. Classical and Quantum Tensor Product Expanders, 2008. arXiv:0804.0011. 
[7] P. Hayden, D. Leung, P. W. Shor, and A. Winter. Randomizing Quantum States: Constructions and Applications. Communications in Mathematical Physics, 250:371-391, 2004. arXiv:quant-ph/0307104.

[8] S. Hoory and A. Brodsky. Simple Permutations Mix Even Better, 2004. arXiv:math/0411098.

[9] M. Kassabov. Symmetric Groups and Expanders, 2005. arXiv:math/0503204.

[10] A. Yu. Kitaev, A. H. Shen, and M. N. Vyalyi. Classical and Quantum Computation. American Mathematical Society, Boston, MA, USA, 2002.

[11] G.-C. Rota. On the foundations of combinatorial theory I. Theory of Möbius Functions . Probability Theory and Related Fields, 2(4):340-368, 1964.

[12] P. Sen. Random measurement bases, quantum state distinction and applications to the hidden subgroup problem. Complexity '06, pages 274-287, 2005. arXiv:quant-ph/0512085.

[13] R. Stanley. Enumerative Combinatorics. Cambridge University Press, Cambridge, UK, 1986. 